\section{Nematode Genome Announcement: Draft Genome of Meloidogyne chitwoodi, an Economically Important Pest of Potato in the Pacific Northwest}

\author{
Sapinder Bali, ${ }^{1,2}$ Shengwei Hu, ${ }^{1}$ Kelly Vining, ${ }^{3}$ Chuck Brown, ${ }^{4}$ Hassan Mojtahedi, ${ }^{4}$ \\ Lei Zhang, ${ }^{2}$ Cynthia Gleason, ${ }^{2}$ and Vidyasagar Sathuvalli ${ }^{1, \dagger}$ \\ ${ }^{1}$ Hermiston Agricultural Research and Extension Station, Oregon State University, Hermiston, OR \\ 19838 \\ ${ }^{2}$ Department of Plant Pathology, Washington State University, Pullman, WA 99164 \\ ${ }^{3}$ Department of Horticulture, Oregon State University, Corvallis, OR 97331 \\ ${ }^{4}$ Retired from United States Department of Agriculture, Prosser, WA 99350
}

\begin{abstract}
Meloidogyne chitwoodi is one of the most devastating pests of potato in the U.S. Pacific Northwest (PNW). Nematode-infected tubers develop external as well as internal defects, making the potato tubers unmarketable, and resulting in economic losses. Draft genome assemblies of three $M$. chitwoodi genotypes-race 1, race 2 and race 1 pathotype Roza-were generated using Illumina and PacBio Sequel RS II sequencing. The final assemblies consist of 30,39 , and 38 polished contigs for race 1 , race 2 and race 1 pathotype Roza, respectively, with average $\mathrm{N}_{50}$ of $2.37 \mathrm{Mb}$ and average assembled genome size of approximately $47.41 \mathrm{Mb}$. On average, 10,508 genes were annotated for each genome. Benchmarking universal single-copy ortholog (BUSCO) analysis indicated that $69.80 \%$ of the BUSCOs were complete whereas $68.80,0.93$, and $12.67 \%$ were single copy, duplicated, and fragmented, respectively. These highly contiguous genomes will enrich resources to study potato-nematode interactions and enhance breeding efforts to develop nematode-resistant potato varieties for the PNW.
\end{abstract}

Potato is one of the major crops of the Pacific Northwest (PNW) region of the United States. Idaho, Oregon, and Washington account for approximately $65 \%$ of the processing and the fresh market potato production in the United States. Meloidogyne chitwoodi Golden et al., commonly known as the Columbia root-knot nematode (CRKN), is an important soilborne, sedentary endoparasite of potato. It was first reported in the Columbia basin in 1980s and, since then, has become a quarantined pest of potato. Apart from potato, M. chitwoodi can also infect tomato, sugar beet, wheat, and corn (Santo et al. 1980). It has a short life cycle (23 to 25 days), resulting in rapid population density build-up over a single cropping season. Second-stage juveniles, the only infective stage in its life cycle, enter the host root tissue and establish feeding sites in the inner cortex region, where they undergo three additional molts and become adult males or females. Mature females produce 800 to 1,000 eggs and lay them in a gelatinous sac on the outer surface of the roots. The peculiarity of nematode infestation in the potato crop is that, in addition to roots, CRKN infects potato tubers.

${ }^{\dagger}$ Corresponding author: V. Sathuvalli; vidyasagar@oregonstate.edu

*The $e$-Xtra logo stands for "electronic extra" and indicates that a supplementary table and supplementary figure are published online.

The author(s) declare no conflict of interest.

Accepted for publication 24 March 2021.
$e-\mathbf{X t r a}^{*}$

Keywords

Columbia root-knot nematode, draft genome, Illumina, PacBio, pathotype, races 
Infected tubers develop internal and external defects, making them unsuitable for fresh market as well as the processing industry. At present, nematode populations are mostly controlled by soil fumigation before the planting season and in-season application of nematicides. Commercial potato varieties resistant to $M$. chitwoodi are unavailable.

Previously, alfalfa was used as a rotation crop to control $M$. chitwoodi populations because the nematode could not reproduce on it (Santo et al. 1980) but, in 1985, one population was found to reproduce on 54 alfalfa accessions and was designated as Alfalfa race or race 2. The populations that did not reproduce on alfalfa were designated as race 1 (Santo and Pinkerton 1985). A later population distribution study in the PNW showed that both race 1 and race 2 coexisted in the majority of potato fields in the region (Pinkerton et al. 1987). Both race 1 and race 2 are unable to reproduce on a diploid wild potato species, Solanum bulbocastanum clone SB22. However, a newly emerged race 1 population identified in Prosser, WA could reproduce on S. bulbocastanum (SB22). This resistance-breaking population was designated as the pathotype of race 1 (Roza) (Mojtahedi et al. 2007).

Sequencing of nematode genomes could offer new tools to study genomics, evolution, and parasitism aspects of the pest. Nematode genomes are relatively small; the size typically ranges between 50 and $250 \mathrm{Mb}$ and for Meloidogyne spp. and the genome size varies between approximately 47 and $51 \mathrm{Mb}$ (Leroy et al. 2003). Genomes of economically important root-knot nematode species have been sequenced in the recent past using various sequencing platforms (Abad et al. 2008; Blanc-Mathieu et al. 2017; Koutsovoulos et al. 2020; Lunt et al. 2014; Opperman et al. 2008; Phan et al. 2020; Sato et al. 2018; Somvanshi et al. 2018; Susič et al. 2020; Szitenberg et al. 2017) (Table 1).

In the present study, we extracted $M$. chitwoodi race 1, race 2, and race 1 (Roza) eggs from 3-month-old infected tomato plants (S. lycopersicum 'Rutgers') using the hypochlorite method (Hussey 1973). Genomic DNA was isolated using a silica-membrane-based nucleic acid purification kit QIAamp DNA Mini Kit (catalog number 51304; Qiagen) following the kit protocol. In total, 30,000 eggs yielded $>2.5 \mu \mathrm{g}$ of DNA, which was sent to the Center for Genome Research and Biocomputing (CGRB), Oregon State University, Corvallis, OR for Illumina sequencing. Two replicate samples of each nematode genotype were barcoded, and sequencing libraries were pooled prior to running on a single lane of a HiSeq3000 (2 $\times$ $150 \mathrm{bp})$. In parallel, libraries from each genotype were also run separately on a MiSeq instrument $(2 \times 350 \mathrm{bp})$.

HiSeq3000 generated an average of 36 billion reads, whereas MiSeq generated 7 million reads on an average for three of the genomes. The quality of the data were checked using FASTQC, and the adapters were filtered and sequences quality trimmed using Cutadapt v. 3.1. The per-base sequence quality of Illumina reads was above average (Supplementary Fig. S1). The microbial and mitochondrial genome contamination was checked during the sequence vetting process done by NCBI servers and removed before final genome submission. The $k$-mer-based estimation of the genome size was calculated using Jellyfish and GenomeScope (Marçais and Kingsford 2011; Vurture et al 2017). Using the cleaned Illumina reads, first, Jellyfish was used to extract and count canonical $k$-mers at $\mathrm{k}=$ 21, 31, and 71 nucleotides. Subsequently, for each k-mer value, GenomeScope was used to estimate heterozygosity, haploid genome length, repeat content, unique length, and read error rate (Supplementary Table S1). The haploid genome size was estimated to be 43.03, 42.68 , and $42.66 \mathrm{Mb}$ for race 1 , race 2 , and Roza, respectively, at $k$-mer 21 (Supplementary Table S1), which is closer to the expected genome size recently reported for $M$. gramninicola (i.e., $41.5 \mathrm{Mb}$ ) (Phan et al. 2020). M. graminicola is closely related to M. chitwoodi because both are placed in clade III based on their molecular phylogenetics.

Highly repetitive nematode genomes challenge the generation of high-quality sequence data using short-read Illumina sequencing platforms. In order to improve the genome contiguity, we complemented the Illumina assembly by resequencing three of the nematode genomes using PacBio Sequel RS II (Pacific Biosciences). This technology is highly efficient and results in longer read lengths, greater consensus accuracy, and a lower degree of sequencing bias (Nakano et al. 2017).

For good-quality, higher molecular weight nematode DNA extraction, approximately $30,000 \mathrm{M}$. chitwoodi eggs each of race 1, race 2, and race 1 (Roza) were sent to Amplicon Expression, and DNA was later sent to CGRB for long-read sequencing. Libraries were prepared using the SMRT bell library prep protocol (Pacific Biosciences), followed by DNA size 
Table 1. Summary of all root-knot nematode genomes sequenced thus far

\begin{tabular}{|c|c|c|c|c|c|c|c|c|c|}
\hline $\begin{array}{l}\text { Nematode } \\
\text { species }\end{array}$ & $\begin{array}{l}\text { Mode of } \\
\text { reproduction }\end{array}$ & Occurrence & $\begin{array}{l}\text { Isolate, strain, } \\
\text { race, pathotype, } \\
\text { genotype }\end{array}$ & $\begin{array}{l}\text { Sequencing } \\
\text { platform }\end{array}$ & $\begin{array}{l}\text { Number of } \\
\text { contigs per } \\
\text { scaffold }\end{array}$ & $\begin{array}{l}\text { Assembly } \\
\text { size } \\
\text { (Mb) }\end{array}$ & $\% G C$ & References & Resources \\
\hline \multirow[t]{3}{*}{$\begin{array}{r}\text { Meloidogyne } \\
\text { chitwoodi }\end{array}$} & $\begin{array}{r}\text { Facultative, meiotic } \\
\text { parthenogenetic }\end{array}$ & $\begin{array}{l}\text { Asia, Africa, } \\
\text { North America, } \\
\text { South America, } \\
\text { Europe }\end{array}$ & PNW-race 1 & $\begin{array}{l}\text { HiSeq } 3000 \\
\text { MiSeq and } \\
\text { PacBio } \\
\text { Sequel RS II }\end{array}$ & 30 & 47.47 & 25 & Present study & PRJNA666745 \\
\hline & - & - & PNW-race 2 & - & 39 & 46.98 & 24.9 & - & - \\
\hline & - & - & PNW-race 1-Roza & - & 38 & 47.78 & 25 & - & - \\
\hline \multirow[t]{2}{*}{ M. graminicola } & $\begin{array}{r}\text { Facultative, meiotic } \\
\text { parthenogenetic }\end{array}$ & $\begin{array}{l}\text { Asia, Africa, } \\
\text { North America, } \\
\text { South America, } \\
\text { Europe }\end{array}$ & IARI strain & Illumina GAllx & 4,304 & 38.18 & 23.1 & $\begin{array}{l}\text { Somvanshi } \\
\text { et al. } 2018\end{array}$ & $\frac{\text { NXFT00000000, }}{\text { SAMN04041660 }}$ \\
\hline & - & - & Mg-VN18 & $\begin{array}{l}\text { Oxford Nanopore } \\
\text { and Illumina }\end{array}$ & 283 & 41.5 & 23.28 & $\begin{array}{l}\text { Phan et al. } \\
2020\end{array}$ & $\begin{array}{l}\text { PRJNA615787, } \\
\text { JABEBT000000000 }\end{array}$ \\
\hline \multirow[t]{3}{*}{ M. arenaria } & $\begin{array}{l}\text { Obligatory, mitotic } \\
\text { parthenogenetic }\end{array}$ & $\begin{array}{l}\text { Asia, Africa, } \\
\text { North America, } \\
\text { Central America } \\
\text { and Caribbean, } \\
\text { South America, } \\
\text { Europe, } \\
\text { Oceania }\end{array}$ & $\mathrm{A} 2-\mathrm{O}$ & $\begin{array}{l}\text { PacBio RS II } \\
\text { platform }\end{array}$ & 2,224 & 284.05 & 30 & $\begin{array}{l}\text { Sato et al. } \\
2018\end{array}$ & QEUI00000000 \\
\hline & - & - & HarA & Illumina & 46,509 & 163.7 & 30.3 & $\begin{array}{l}\text { Szitenberg } \\
\text { et al. } 2017\end{array}$ & PRJNA340324 \\
\hline & - & - & Guadeloupe & $\begin{array}{l}\text { 454, Illumina } \\
\text { HiSeq } 2000\end{array}$ & 26,196 & 258.07 & 29.8 & $\begin{array}{c}\text { Blanc-Mathieu } \\
\text { et al. } 2017\end{array}$ & PRJEB871 \\
\hline \multirow[t]{2}{*}{ M. javanica } & $\begin{array}{l}\text { Obligatory, mitotic } \\
\text { parthenogenetic }\end{array}$ & $\begin{array}{l}\text { Asia, Africa, } \\
\text { North America, } \\
\text { Central America } \\
\text { and Caribbean, } \\
\text { South America, } \\
\text { Europe, } \\
\text { Oceania }\end{array}$ & Avignon & $\begin{array}{l}\text { 454, Illumina } \\
\text { HiSeq } 2000\end{array}$ & 31,341 & 235.8 & 29.9 & $\begin{array}{c}\text { Blanc-Mathieu } \\
\text { et al. } 2017\end{array}$ & PRJEB871 \\
\hline & - & - & VW4 & Illumina & 34,394 & 142.6 & 30.2 & $\begin{array}{l}\text { Szitenberg } \\
\text { et al. } 2017\end{array}$ & PRJNA340324 \\
\hline \multirow[t]{3}{*}{ M. incognita } & $\begin{array}{l}\text { Obligatory, mitotic } \\
\text { parthenogenetic }\end{array}$ & $\begin{array}{l}\text { Asia, Africa, } \\
\text { North America, } \\
\text { Central America } \\
\text { and Caribbean, } \\
\text { South America, } \\
\text { Europe, } \\
\text { Oceania }\end{array}$ & Morelos (INRA) & $\begin{array}{l}\text { 454, Illumina } \\
\text { HiSeq } 2000\end{array}$ & 12,091 & 183.53 & 29.8 & $\begin{array}{c}\text { Blanc-Mathieu } \\
\text { et al. } 2017\end{array}$ & PRJEB871 \\
\hline & - & - & W1 & Illumina & 33,735 & 122 & 30.6 & $\begin{array}{l}\text { Szitenberg } \\
\text { et al. } 2017\end{array}$ & PRJNA340324 \\
\hline & - & - & Morelos (INRA) & $\begin{array}{l}\text { Sanger } \\
\qquad \text { ABI3730X1 } \\
\text { DNA Analyzer }\end{array}$ & 2,817 & 86 & 31.4 & $\begin{array}{l}\text { Abad et al. } \\
2008\end{array}$ & САBB01000001 \\
\hline \multirow[t]{2}{*}{ M. floridensis } & $\begin{array}{r}\text { Facultative, meiotic } \\
\text { parthenogenetic }\end{array}$ & $\begin{array}{l}\text { North America } \\
\text { (Florida) }\end{array}$ & SJF1 & Illumina & 9,134 & 74.8 & 30.2 & $\begin{array}{l}\text { Szitenberg } \\
\text { et al. } 2017\end{array}$ & PRJNA340324 \\
\hline & & & Isolate-5 (USA) & $\begin{array}{l}\text { Illumina } \\
\text { HiSeq2000 }\end{array}$ & 81,111 & 99.8 & 30 & Lunt et al. 2014 & ERP001338 \\
\hline \multirow[t]{2}{*}{ M. enterolobii } & $\begin{array}{l}\text { Obligatory, mitotic } \\
\text { parthenogenetic }\end{array}$ & $\begin{array}{l}\text { Asia, Africa, } \\
\text { North America, } \\
\text { Central America } \\
\text { and Caribbean, } \\
\text { South America, } \\
\text { Europe }\end{array}$ & Swiss pop & $\begin{array}{l}\text { Illumina and } \\
\text { PacBio RS II } \\
\text { platform }\end{array}$ & 4437 & 240 & 30 & $\begin{array}{r}\text { Koutsovoulos } \\
\text { et al. } 2020\end{array}$ & $\begin{array}{l}\text { https://meloidogyne. } \\
\text { inrae.fr/. }\end{array}$ \\
\hline & - & - & L30 & Illumina & 46,090 & 162.3 & 30.2 & $\begin{array}{l}\text { Szitenberg } \\
\text { et al. } 2017\end{array}$ & PRJNA340324 \\
\hline M. hapla & $\begin{array}{r}\text { Facultative, meiotic } \\
\text { parthenogenetic }\end{array}$ & $\begin{array}{l}\text { Asia, Africa, } \\
\text { North America, } \\
\text { Central America } \\
\text { and Caribbean, } \\
\text { South America, } \\
\text { Europe, } \\
\text { Oceania }\end{array}$ & VW9 & $\begin{array}{c}\text { ABI3730, } \\
\text { Megabase } \\
\text { Sequence } \\
\text { Analyzer }\end{array}$ & 1,523 & 54 & 27.4 & $\begin{array}{l}\text { Opperman } \\
\text { et al. } 2008\end{array}$ & SAMN02743742 \\
\hline M. luci & $\begin{array}{l}\text { Obligatory, mitotic } \\
\text { parthenogenetic }\end{array}$ & $\begin{array}{c}\text { Asia, Europe (few } \\
\text { occurrences), } \\
\text { North America, } \\
\text { South America }\end{array}$ & SI-Smartno-V13 & $\begin{array}{c}\text { PacBio RS II } \\
\text { platform }\end{array}$ & 327 & 209.16 & 30.2 & $\begin{array}{l}\text { Susič et al. } \\
2020\end{array}$ & ERS3574357 \\
\hline
\end{tabular}

selection using the Blue Pippin system (Sage Science) to remove short insert templates. Subsequently, M. chitwoodi DNA was sequenced on PacBio Sequel RS II (Pacific Biosciences). Genomes were assembled using the SMRT analysis pipeline, v.2.3.0. Illumina reads were used to error correct PacBio data contigs using pilon v.1.22 (Walker et al. 2014). The final sequence assembly resulted in 30,39 , and 38 highly polished contigs for race 1 , 
Table 2. Summary of statistics of Meloidogyne chitwoodi race 1 (Mc1), race 2 (Mc27), and race 1 pathotype (Roza) sequenced genomes

\begin{tabular}{lcccccc} 
Genotype & $\begin{array}{c}\text { Assembly } \\
\text { size } \\
\text { (bp) }\end{array}$ & $\begin{array}{c}\text { Maximum } \\
\text { contig } \\
\text { length (bp) }\end{array}$ & $\begin{array}{c}\mathbf{N}_{\mathbf{5 0}} \text { contig } \\
\text { length } \\
\text { (bp) }\end{array}$ & $\begin{array}{c}\text { Number of } \\
\text { polished } \\
\text { contigs }\end{array}$ & $\begin{array}{c}\text { Estimated } \\
\text { genome } \\
\text { coverage (x) }\end{array}$ & $\begin{array}{c}\text { Haploid } \\
\text { genome } \\
\text { size (Mb) }\end{array}$ \\
Mc1 & $47,477,280$ & $3,043,654$ & $2,451,023$ & 30 & 172.04 & 43.03 \\
Mc27 & $46,924,610$ & $3,043,255$ & $2,317,798$ & 39 & 84.34 & 42.68 \\
Roza & $47,730,107$ & $2,942,887$ & $2,363,161$ & 38 & 168.05 & 42.66 \\
\hline
\end{tabular}

${ }^{a}$ Estimation ( $k$-mer based) calculated using Jellyfish and GenomeScope ( $k$-mer $=21$ nucleotides).

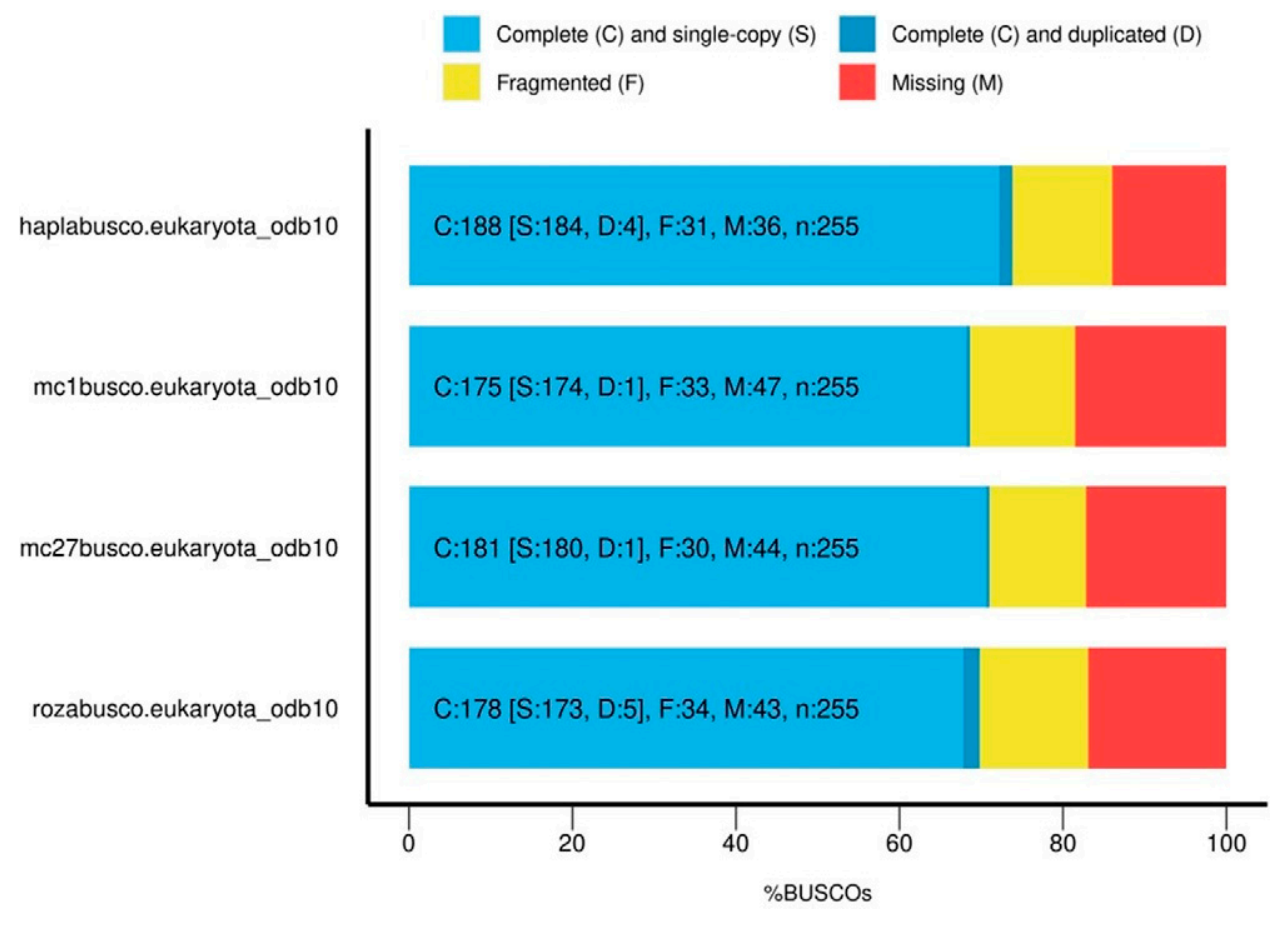

Fig. 1. Summary of benchmarking universal single-copy ortholog (BUSCO) score analysis calculated for Meloidogyne chitwoodi race 1 (Mc1), race 2 (Mc27), and race 1 pathotype (Roza) sequenced genomes using the eukaryota database. M. hapla was used as a control and reference point.

Table 3. Benchmarking universal single-copy ortholog (BUSCO) score estimation of Meloidogyne chitwoodi race 1 (Mc1), race 2 (Mc27), and race 1 pathotype (Roza) sequenced genomes as compared with publicly available $M$. hapla genome ${ }^{a}$

\begin{tabular}{lccccc}
$\begin{array}{l}\text { Name } \\
\text { of the }\end{array}$ & $\begin{array}{c}\text { Complete } \\
\text { BUSCOs (C) } \\
\text { nematode }\end{array}$ & $\begin{array}{c}\text { Complete and } \\
\text { single-copy } \\
\text { BUSCOs (S) }\end{array}$ & $\begin{array}{c}\text { Complete and } \\
\text { duplicated } \\
\text { BUSCOs (D) } \\
(\%)\end{array}$ & $\begin{array}{c}\text { Fragmented } \\
\text { BUSCOs (F) }\end{array}$ & $\begin{array}{c}\text { Missing } \\
\text { BUSCOs (M) } \\
\text { (\%) }\end{array}$ \\
Mc1 & 68.6 & 68.2 & 0.4 & 12.9 & 18.5 \\
Mc27 & 71.0 & 70.6 & 0.4 & 11.8 & 17.2 \\
Roza & 69.8 & 67.8 & 2.0 & 13.3 & 16.9 \\
M. hapla & 73.8 & 72.2 & 1.60 & 12.2 & 14.0 \\
\hline
\end{tabular}

a Scores were calculated using eukaryote odb10 dataset (BUSCO v5.0.0).

race 2, and race 1 (Roza), respectively. The sum of all contig lengths was $47.47 \mathrm{Mb}$ for race 1 ( $n=30$ contig lengths), $46.98 \mathrm{Mb}$ for race $2(n=39)$, and $47.78 \mathrm{Mb}$ for race 1 (Roza) ( $n=38$ ) (Table 2). The largest contig length was $3.04 \mathrm{Mb}$ for race 1, $3.04 \mathrm{Mb}$ for race 2, and $2.94 \mathrm{Mb}$ for race 1 (Roza). All three nematode genomes sequenced in the present study contained an average of $25 \% \mathrm{GC}$ content. 
Genome annotation was performed using the $M$. hapla protein dataset from wormBase and the SNAP training file prepared from the first round of annotation. The annotated gene numbers for race 1 , race 2 , and race 1 (Roza) were 10,441, 10,424, and 10,660, respectively. To check the completeness of the genomes, benchmarking universal single-copy ortholog (BUSCO) scores were calculated using the lineage dataset, eukaryota_odb10 (with number of species $=70$ ). On average, three of the genomes showed $69.80 \%$ complete, $68.80 \%$ single copy, $0.93 \%$ duplicated, $12.67 \%$ fragmented, and $17.53 \%$ missing BUSCOs and are similar to the published $M$. hapla genome (Fig. 1; Table 3).

The whole-genome project has been deposited at DNA Data Bank of Japan/European Nucleotide Archive/GenBank under the BioProject number PRJNA666745. Three of the genomes can be accessed under accession numbers JACZZP000000000 (Mc1), JACZZO000000000 (Mc2), and JACZZN000000000 (Roza). Raw data files and the genome annotations are available at the Sathuvalli Lab website.

To the best of our knowledge, this is the first ever report of draft genomes of the $M$. chitwoodi races and pathotype prevalent in the PNW. The sequencing of highly contagious $M$. chitwoodi genomes will enable the identification of effectors involved in the susceptibility mechanism which, in turn, will enhance our knowledge of host-pathogen interactions. In addition, the sequence data could be used to design DNA-based markers for the identification and differentiation of $M$. chitwoodi races and pathotypes for diagnostic purposes. The availability of high-quality reference genomes for $M$. chitwoodi races and pathotypes will be a major step in understanding the nematode's evolution and diversification.

\section{Author-Recommended Internet Resources}

wormBase: https://www.wormbase.org

Sathuvalli Lab website: http://files.cgrb.oregonstate.edu/Sathuvalli_Lab/

Genome annotations: http://files.cgrb.oregonstate.edu/Sathuvalli_Lab/Meloidogyne_chitwoodi_GenomeAnnotation

\section{Literature Cited}

Abad, P., Gouzy, J., Aury, J. M., Castagnone-Sereno, P., Danchin, E. G., Deleury, E., Perfus-Barbeoch, L., Anthouard, V., Artiguenave, F., Blok, V. C., Caillaud, M. C., Coutinho, P. M., Dasilva, C., De Luca, F., Deau, F., Esquibet, M., Flutre, T., Goldstone, J. V., Hamamouch, N., Hewezi, T., Jaillon, O., Jubin, C., Leonetti, P., Magliano, M., Maier, T. R., Markov, G. V., McVeigh, P., Pesole, G., Poulain, J., Robinson-Rechavi, M., Sallet, E., Ségurens, B., Steinbach, D., Tytgat, T., Ugarte, E., van Ghelder, C., Veronico, P., Baum, T. J., Blaxter, M., Bleve-Zacheo, T., Davis, E. L., Ewbank, J. J., Favery, B., Grenier, E., Henrissat, B., Jones, J. T., Laudet, V., Maule, A. G., Quesneville, H., Rosso, M. N., Schiex, T., Smant, G., Weissenbach, J., and Wincker, P. 2008. Genome sequence of the metazoan plant-parasitic nematode Meloidogyne incognita. Nat. Biotechnol. 26:909-915.

Blanc-Mathieu, R., Perfus-Barbeoch, L., Aury, J. M., Da Rocha, M., Gouzy, J., Sallet, E., Martin-Jimenez, C., Bailly-Bechet, M., Castagnone-Sereno, P., Flot, J. F., Kozlowski, D. K., Cazareth, J., Couloux, A., Da Silva, C., Guy, J., Kim-Jo, Y. J., Rancurel, C., Schiex, T., Abad, P., Wincker, P., and Danchin, E. G. J. 2017. Hybridization and polyploidy enable genomic plasticity without sex in the most devastating plant-parasitic nematodes. PLoS Genet. 13:e1006777.

Hussey, R. S. 1973. A comparison of methods of collecting inocula of Meloidogyne spp., including a new technique. Plant Dis. Rep. 57:1025-1028.

Koutsovoulos, G. D., Poullet, M., Elashry, A., Kozlowski, D. K. L., Sallet, E., Da Rocha, M., Perfus-Barbeoch, L., Martin-Jimenez, C., Frey, J. E., Ahrens, C. H., Kiewnick, S., and Danchin, E. G. J. 2020. Genome assembly and annotation of Meloidogyne enterolobii, an emerging parthenogenetic rootknot nematode. Sci. Data 7:324.

Leroy, S., Duperray, C., and Morand, S. 2003. Flow cytometry for parasite nematode genome size measurement. Mol. Biochem. Parasitol. 128:91-93.

Lunt, D. H., Kumar, S., Koutsovoulos, G., and Blaxter, M. L. 2014. The complex hybrid origins of the root knot nematodes revealed through comparative genomics. PeerJ 2:e356.

Marçais, G., and Kingsford, C. 2011. A fast, lock-free approach for efficient parallel counting of occurrences of $k$-mers. Bioinformatics 27:764-770.
Mojtahedi, H., Brown, C. R., Riga, E., and Zhang, L. H. 2007. A new pathotype of Meloidogyne chitwoodi race 1 from Washington State. Plant Dis. 91:1051.

Nakano, K., Shiroma, A., Shimoji, M., Tamotsu, H., Ashimine, N., Ohki, S., Shinzato, M., Minami, M., Nakanishi, T., Teruya, K., Satou, K., and Hirano, T. 2017. Advantages of genome sequencing by long-read sequencer using SMRT technology in medical area. Hum. Cell 30:149-161.

Opperman, C. H., Bird, D. M., Williamson, V. M., Rokhsar, D. S., Burke, M., Cohn, J., Cromer, J., Diener, S., Gajan, J., Graham, S., Houfek, T. D., Liu, Q., Mitros, T., Schaff, J., Schaffer, R., Scholl, E., Sosinski, B. R., Thomas, V. P., and Windham, E. 2008. Sequence and genetic map of Meloidogyne hapla: A compact nematode genome for plant parasitism. Proc. Natl. Acad. Sci. U.S.A. 105:14802-14807.

Phan, N. T., Orjuela, J., Danchin, E. G. J., Klopp, C., Perfus-Barbeoch, L., Kozlowski, D. K., Koutsovoulos, G. D., Lopez-Roques, C., Bouchez, O., Zahm, M., Besnard, G., and Bellafiore, S. 2020. Genome structure and content of the rice root-knot nematode (Meloidogyne graminicola). Ecol. Evol. 10:11006-11021.

Pinkerton, J. N., Mojtahedi, H., and Santo, G. S. 1987. Reproductive efficiency of Pacific Northwest isolates of Meloidogyne chitwoodi on alfalfa. Plant Dis. 71:345-348.

Santo, G. S., O'Bannon, J. H., and Finley, A. M. 1980. Occurrence and host range of a new root-knot nematode species (Meloidogyne chitwoodl) in the Pacific Northwest. Plant Dis. 64:951-952.

Santo, G. S., and Pinkerton, J. N. 1985. A second race of Meloidogyne chitwoodi discovered in Washington. Plant Dis. 69:631.

Sato, K., Kadota, Y., Gan, P., Bino, T., Uehara, T., Yamaguchi, K., Ichihashi, Y., Maki, N., Iwahori, H., Suzuki, T., Shigenobu, S., and Shirasu, K. 2018. Highquality genome sequence of the root-knot nematode Meloidogyne arenaria genotype A2-O. Genome Announce. 6:e00519-18.

Somvanshi, V. S., Tathode, M., Shukla, R. N. and Rao, U. 2018. Nematode genome announcement: A draft genome for rice root-knot nematode, Meloidogyne graminicola. J. Nematol. 50:111-116. 
Susič, N., Koutsovoulos, G. D., Riccio, C., Danchin, E. G. J., Blaxter, M. L., Lunt, D. H., Strajnar, P., Šrca, S., Urek, G., and Stare, B. G. 2020. Genome sequence of the root-knot nematode Meloidogyne luci. J. Nematol. 52:e2020-25.

Szitenberg, A., Salazar-Jaramillo, L., Blok, V. C., Laetsch, D. R., Joseph, S., Williamson, V. M., Blaxter, M. L., and Lunt, D. H. 2017. Comparative genomics of apomictic root-knot nematodes: Hybridization, ploidy, and dynamic genome change. Genome Biol Evol. 9:2844-2861.
Vurture, G. W., Sedlazeck, F. J., Nattestad, M., Underwood, C. J., Fang, H., Gurtowski, J., and Schatz, M. C. 2017. GenomeScope: Fast reference-free genome profiling from short reads. Bioinformatics 33:2202-2204.

Walker, B. J., Abeel, T., Shea, T., Priest, M., Abouelliel, A., Sakthikumar, S., Cuomo, C. A., Zeng, Q., Wortman, J., Young, S. K., and Earl, A. M. 2014. Pilon: An integrated tool for comprehensive microbial variant detection and genome assembly improvement. PLoS One 9:e112963. 